\section{Surgical resection for pulmonary metastases of sweat gland carcinoma}

Toshihiro Osaki, Mantaro Kodate, Ryoichi Nakanishi, Tetsuya Mitsudomi, Takayuki Shirakusa

\begin{abstract}
A case of axillary sweat gland carcinoma which metastasised to both lungs six years after resection of the primary lesion is described. The lesions were resected and three years later two further pulmonary metastases were resected.
\end{abstract}

(Thorax 1994;49:181-182)

Sweat gland carcinoma is very rare with a reported incidence of less than $0.005 \%$ of all tumour specimens resected surgically. ${ }^{1}$ Nearly all sweat gland carcinomas are treated satisfactorily by local excision. ${ }^{1}$ The predominant route of metastatic spread is by the lymphatics but in $48 \%$ of the cases there was haematogenous spread. ${ }^{23}$ When distant metastases occur they are generally considered to be resistant to radiotherapy and chemotherapy. We report a case of axillary sweat gland carcinoma with bilateral pulmonary metastases in which surgical resection was performed three times over three years.

\section{Case report}

In March 1982 a 50 year old Japanese man underwent local excision of a sweat gland carcinoma of the right axilla. Neither chemotherapy nor radiation were administered. Six years later the patient was referred with metastatic round lesions on the chest radiograph, two on the left and one on the right (fig 1). At that time there were no palpable lymph nodes, no clinical recurrence at the original site, and no distant metastases. Further investigation provided no evidence for another primary malignancy. In July 1988 wedge resection of the metastasis in the right lung was performed followed by wedge resection of the lesions in the left lung in October 1988. In October 1991 two further lesions developed in the left lung which were also resected by segmental excision.

The excised specimen showed histopathological features similar to the previous specimens. Routine hematoxylin and eosin stained paraffin sections of the lung metastases showed that the tumour consisted of papillotubular formations of atypical glandular cells with eosinophilic cytoplasm, with a cribriform pattern in parts. In some areas "decapitation" secretion was seen. Stains for ferrum were negative. Electron microscopic findings showed nuclei with marked indentations and the cytoplasm was abundant in rough endoplasmic reticulum. Microvilli projecting into the lumen, tight junctions at the cell borders involving desmosomes, and active "decapitation" secretion was seen (fig 2). This tumour appeared to be a sweat gland carcinoma arising from the apocrine sweat apparatus.

Ten years after resection of the axillary tumour the patient is alive and well, with no evidence of further recurrent disease.

\section{Discussion}

Sweat gland carcinomas are rare malignant tumours that were first described by Cornil in $1865 .^{4}$ The variability of the histological features even in the same tumour, and its rarity, have contributed to some confusion regarding the classification of sweat gland carcinoma. The histological classification put forward by Lever and Schaumburg-Lever ${ }^{5}$ includes a classic type, mucinous (adenocystic) carcinoma, adenoid cystic carcinoma, syringoid eccrine carcinoma, microcystic adnexal carcinoma, aggressive digital papillary adenocarcinoma, and carcinoma of apocrine glands. In the present case the histological features coincided with a carcinoma of the apocrine glands which consisted of atypical glandular cells with eosinophilic cytoplasm and active "decapitation" secretion. S100 protein and carcinoembryonic antigen have been widely used as immunohistochemical markers for various kinds of tumours including sweat gland neoplasms. ${ }^{56}$ Clinicohistologically, sweat gland carcinoma has to be primarily differentiated from metastatic carcinomas such as those of the breast, kidney, visceral organs, salivary gland, and nasopharynx. ${ }^{5}$ In our case intensive investigation failed to disclose any origin of the tumour other than the skin of the right axilla.

Sweat gland carcinoma has a chronic and

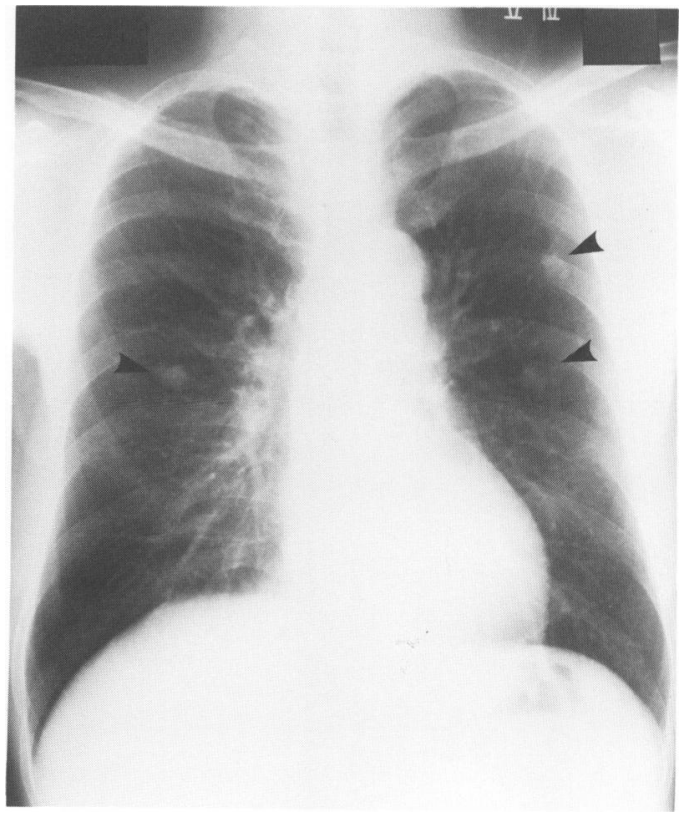

Figure 1 Chest radiograph showing metastatic round lesions, two on the left and one on the right. 


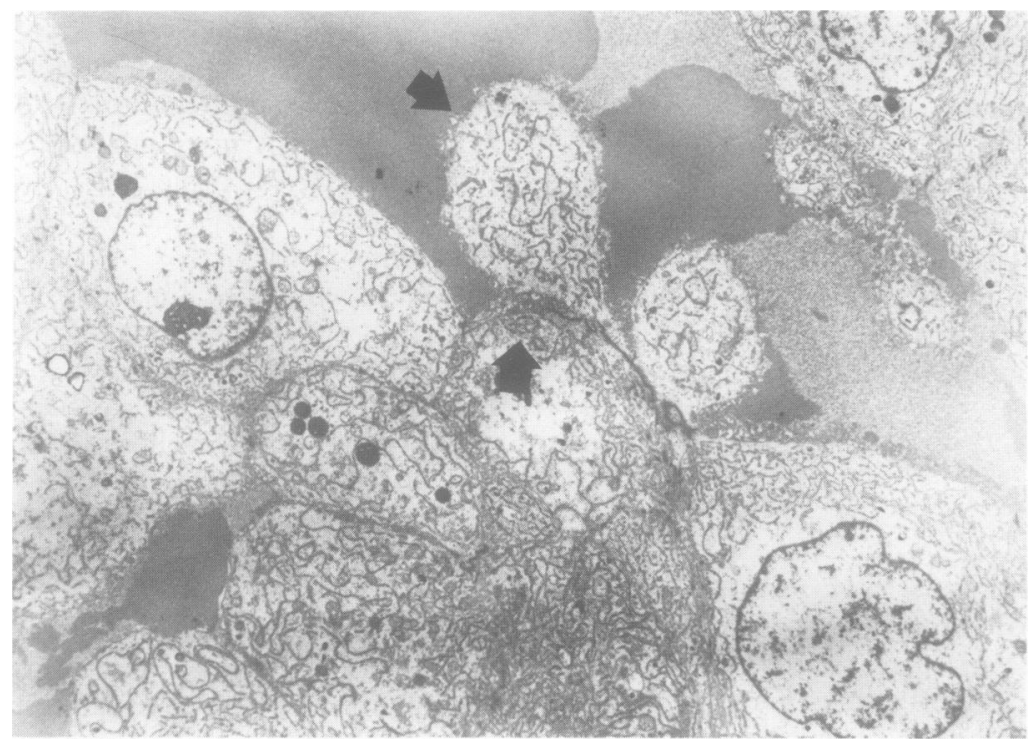

Figure 2 Electron micrograph showing "decapitation" secretion (arrows). This tumour appeared to be a sweat gland carcinoma arising from the apocrine sweat glands. Original magnification $\times 2000$, reduced to $60 \%$ in origination.

protracted course. ${ }^{3}$ It is possible to separate these tumours histologically into low and high grade variants. This separation correlates with the frequency of metastases and local recurrence. El-Domeiri $e t a l^{3}$ noted that $70 \%$ of patients with low grade sweat gland carcinoma were free of disease after five years while none of those with a high grade undifferentiated tumour were alive at five years. When distant metastases occur they are associated with a poor prognosis. Coonley et al reported that, in 20 evaluable patients with metastatic sweat gland cancer and several trials of chemotherapy, only two partial responses and two minor responses were obtained. Whittington $e t$ $a l^{8}$ reviewed 17 reported experiences with radiation and stated that the outcome was poor. It is not clear whether the poor results reported previously are due to true resistance of the tumour to radiation and chemotherapy, or to the dose and field size of radiation, or to inadequate available chemotherapeutic regimens and agents.

The treatment for metastatic disease is not defined, however, and surgery may offer some benefit. In most instances when pulmonary metastases are found, there are other distant lesions present and surgery is inappropriate. There are few reports of surgical treatment for sweat gland metastases to the lung. Zamboni et $a l^{9}$ reported a case of sweat gland carcinoma with bilateral pulmonary metastases in a patient who underwent a staged left lower lobectomy and wedge resection of the right lung and was free of disease two years later. Although there are not enough cases currently reported to evaluate the treatment of metastatic tumours or the value of palliative resection, our case suggests that patients with a sweat gland carcinoma with lung metastases developing some years after resection of the primary tumour can achieve complete remission by resection of the metastatic lesions.

1 Tulenko JF, Conway H. An analysis of sweat gland tumours. Surg Gynecol Obstet 1965;121:343-8.

2 Hirsh LF, Enterline HT, Rosato EF, Rosato FE. Sweat gland carcinoma. Ann Surg 1971;174:283-6.

3 El-Domeiri AA, Brasfield RD, Huvos AG, Strong EW. Sweat gland carcinoma: a clinicopathological study of 83 patients. Ann Surg 1971;173:270-4.

4 Smith CCK. Metastasizing carcinoma of the sweat glands. Br f Surg 1955;43:80-4.

5 Lever WF, Schaumburg-Lever G. Histopathology of the skin. 7th edn. Philadelphia: Lippincott, 1990:635-9.

6 Penneys NS, Nadji M, Ziegels-Weissman J, Ketabchi M, Morales AR. Carcinoembryonic antigen in sweat gland Morales AR. Carcinoembryonic anti
carcinomas. Cancer 1982;50:1608-11.

7 Coonley CJ, Kelsen DP, Huvos AG, Schauer P, Sordillo P. Chemotherapy of metastatic sweat gland carcinoma. $A m \dot{\mathcal{J}}$ Clin Oncol 1985;8:307-11.

8 Whittington R, Browning ME, Farrell GR, Miremadi A. Radiation therapy and chemotherapy in malignant sweat gland tumors. $\mathcal{F}$ Am Acad Dermatol 1986;15:1093-7.

9 Zamboni AC, Zamboni WA, Ross DS. Malignant eccrine spiradenoma of the hand. $\mathcal{f}$ Surg Oncol 1990;43:131-3. 